\title{
The effect of opacity on the evolution of giant planets
}

\author{
Allona Vazan ${ }^{1}$, Attay Kovetz ${ }^{1,2}$ and Morris Podolak ${ }^{1}$ \\ ${ }^{1}$ Department of Geophysics and Planetary Sciences, Sackler Faculty of Exact Sciences \\ Tel Aviv University, Israel \\ ${ }^{2}$ School of Physics and Astronomy, Sackler Faculty of Exact Sciences \\ Tel Aviv University, Israel \\ email: allonava@post.tau.ac.il
}

\begin{abstract}
We use an improved version of the planetary evolution code described in Helled et al. (2006) to model the effect of opacity on the evolution of giant planets in the disk instability scenario. We find that changing the opacity law can cause significant changes in the evolutionary path of a protoplanet. Sufficiently high opacities cause oscillatory behavior that delays the final collapse. Peak luminosities just before collapse can exceed $10^{-5} L_{\odot}$.
\end{abstract}

Keywords. planets and satellites: formation, instabilities

\section{Introduction}

One of the important parameters in determining the evolutionary path of a protoplanet is the assumed opacity law (e.g. Pollack et al. 1994; D'Alessio et al. 2001). Microphysical processes can cause the opacity law to differ substantially from the interstellar values. The resulting difference has been shown to have important consequences for the evolutionary track of a protoplanet (Hubickyj et al. 2005; Movshovitz et al. 2010).

\section{The Model}

We use a stellar evolution code (Kovetz et al. 2009), that was modified to work under planetary conditions (Helled et al. 2006). We consider giant planet formation in the disk instability scenario (Boss 2002; Mayer et al. 2007; Boley 2009). The initial configuration is a low-density, roughly isentropic, clump of one Jupiter mass, composed of a solar mix of hydrogen and helium. Our standard initial model, kindly provided by P. Bodenheimer (personal communication), has a radius of $101 R_{\odot} \sim 0.5 \mathrm{AU}$. This is roughly equal to the Hill sphere radius.

In order to study the effect of opacity we developed a simple grain opacity code, which assumes a power law size distribution of grains composed of ice and silicates. Although there is no intent to model the details of the chemistry, we do include grain evaporation and the details of Mie scattering for spherical grains. In this way, we can test the importance of such parameters as the grain to gas mass ratio, the ice to silicate mass ratio, and the exponent in the power law size distribution $(\gamma)$. Gas opacities were taken from tables of opacity kindly supplied by A. Burrows (Sharp \& Burrows 2007).

\section{Results}

For a given grain to gas ratio, the opacity will depend strongly on the ratio of grain size to wavelength, and hence on $\gamma$. For low $\gamma$ most of the mass is in large grains, which have 
a low surface area to mass ratio. As $\gamma$ increases the mass is distributed among smaller grains and the opacity increases. For still larger $\gamma$ the mass is concentrated in the smallest grains, which are inefficient scatterers. Fig. 1 shows the evolutionary track of a clump as a function of $\gamma$. For low opacity (low and high $\gamma$ ) the clump cools, becomes dynamically unstable, and collapses to a more compact configuration. For $\gamma$ in the neighborhood of 4 oscillations are seen which significantly extend the contraction stage, and, in some cases no dynamical collapse is seen. The models that eventually collapse show a markedly
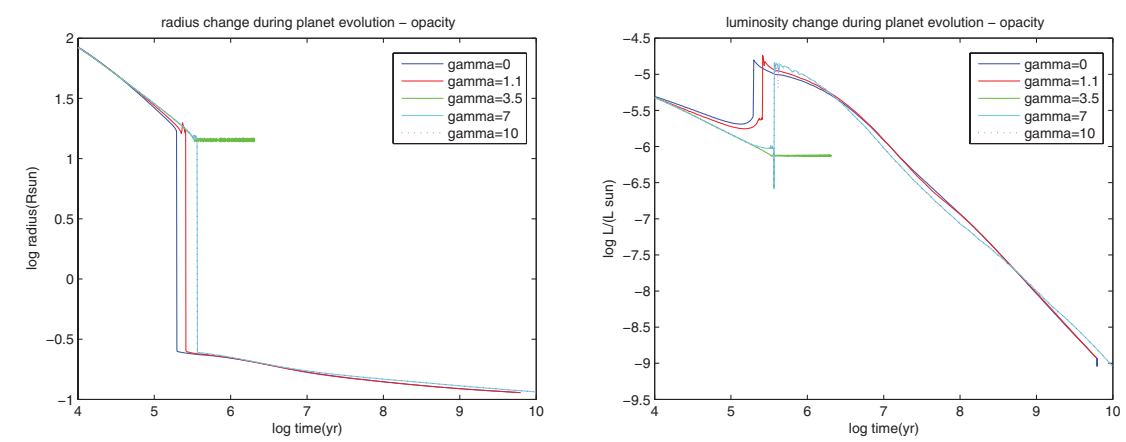

Figure 1. Planetary radius and luminosity as a function of time, for different grain size distributions $(\gamma)$.

lower opacity at the center. This behavior is reminiscent of the $\kappa$-mechanism for stellar pulsations. The actual dynamic collapse, however, appears to be initiated by a radiative core. If the opacity is high enough, there is no such radiative region, and the model appears to settle down, with small oscillations around a quasi-static configuration.

The opacity can be increased, either by raising the mass fraction of grain material, or by concentrating the grain mass at grain sizes where the extinction was most efficient. For sufficiently high values of the opacity, oscillations appear substantially lengthen the contraction time. If the opacity is high enough, the collapse to planetary size could be delayed by millions of years. Delaying the dynamic collapse of an extended protoplanet will allow it to maintain a large cross section for planetesimal capture for a longer time, and this will increase the amount of high-Z material that it can capture and, in turn, increase the grain opacity. A self-consistent calculation of this effect is important, and will be undertaken in future work.

\section{References}

Boss, A. P. 2002, ApJ, 576, 462

Boley, A. C. 2009, ApJL, 695, L53

D'Alessio, P., Calvet, N., \& Hartmann, L. 2001, ApJ, 553, 321

Helled, R., Podolak, M., \& Kovetz, A. 2006, Icarus, 185, 64

Hubickyj, O., Bodenheimer, P., \& Lissauer, J. J. 2005, Icarus, 179, 415

Kovetz, A., Yaron, O., \& Prialnik, D. 2009, MNRAS, 395, 1857

Mayer, L., Lufkin, G., Quinn, T., \& Wadsley, J. 2007, ApJL, 661, L77

Movshovitz, N., Bodenheimer, P., Podolak, M., \& Lissauer, J. J. 2010, Icarus, 209, 616

Pollack, J. B., Hollenbach, D., Beckwith, S., Simonelli, D. P., Roush, T., \& Fong, W. 1994, ApJ, 421,615

Sharp, C. M. \& Burrows, A. 2007, ApJS, 168, 140 